Minoru Asada • Yoshiyuki Okano • Takuji Imamura

Itsujin Suyama • Yutaka Hase • Gen Isshiki

\title{
Molecular characterization of galactokinase deficiency in Japanese patients
}

\begin{abstract}
Galactokinase (GALK) deficiency is an autosomal recessive disorder, which causes cataract formation in children not maintained on a lactose-free diet. We characterized the human $G A L K$ gene by screening a Japanese genomic DNA phage library, and found that several nucleotides in the $5^{\prime}$-untranslated region and introns 1,2 , and 5 in our $G A L K$ genomic analysis differed from published data. A 20-bp tandem repeat was found in three places in intron 5 , which were considered insertion sequences. We identified five novel mutations in seven unrelated Japanese patients with GALK deficiency. There were three missense mutations and two deletions. All three missense mutations (R256W, T344M, and G349S) occurred at CpG dinucleotides, and the T344M and G349S mutations occurred in the conserved region. The three missense mutations led to a drastic reduction in GALK activity when individual mutant cDNAs were expressed in a mammalian cell system. These findings indicated that these missense mutations caused GALK deficiency. The two deletions, of 410delG and 509510delGT, occurred at the nucleotide repeats GGGGGG and GTGTGT, respectively, and resulted in in-frame nonsense codons at amino acids 163 and 201. These mutations arose by slipped strand mispairing. All five mutations occurred at hot spots in the $\mathrm{CpG}$ dinucleotide for missense mutations and in short direct repeats for deletions. These five mutations in Japanese have not yet been identified in Caucasians. We speculate that the origin of GALK mutations in Japanese is different from that in Caucasians.
\end{abstract}

M. Asada $\cdot$ Y. Okano $(\square) \cdot$ T. Imamura $\cdot$ G. Isshiki

Department of Pediatrics, Osaka City University Medical School,

1-4-3 Asahi-machi, Abeno-ku, Osaka 545-8585, Japan

Tel. +81-6-6645-3816; Fax +81-6-6636-8737

e-mail: okano@med.osaka-cu.ac.jp

I. Suyama

Osaka Municipal Rehabilitation Center for the Disabled, Osaka, Japan

Y. Hase

Ikuno Public Health Center of Osaka City, Osaka, Japan
Key words Galactosemia - Galactokinase (GALK) · Mutation - Genotype $\cdot$ Phenotype

\section{Introduction}

Galactokinase (GALK: McKUSICK 230200) is the first enzyme in the Leloir pathway of galactose metabolism; it catalyzes the phosphorylation of galactose to galactose1-phosphate. GALK deficiency, first described in 1965 (Gitzelmann 1965), is an autosomal recessive genetic disorder with an incidence of 1/1,000,000 in Japan (Aoki and Wada 1988) on newborn mass screening and an incidence of $1 / 1,000,000$ in Caucasians (Segal and Berry 1995). It causes mainly cataract formation (Stambolian 1988), galactosemia, galactosuria, and, on rare occasions, pseudotumor cerebri (Litman et al. 1975) and mental retardation (Segal et al. 1979) in newborns exposed to dietary galactose. Cataract is the result of osmotic phenomena caused by the accumulation of galactitol in the lens. The galactitol is synthesized from the reduction of galactose by aldose reductase. It has been suspected that this cataract formation is caused by hypergalactosemia due to the presence of a partial or complete enzyme deficiency in the galactose metabolic pathway, and/or high adult jejunal lactase activity, and/or high consumption of lactose. The partial enzyme deficiency involves heterozygous GALK deficiency and variants of GALK, including Philadelphia (Tedesco et al. 1977) and Urbino variants (Magnani et al. 1982), exhibiting, respectively, 70\% and $50 \%$ of the GALK activity of wild-type controls. However, it remains unclear whether heterozygous GALK deficiency and variants cause presenile cataract formation. Molecular genetic analysis of the $G A L K$ gene will contribute to the investigation of GALK deficiency and to elucidating the effect of galactose metabolism on cataract formation.

cDNA encoding human GALK was cloned and functionally characterized in 1995 (Stambolian et al. 1995); it was $1.35 \mathrm{~kb}$ in length and encoded a peptide of 392 amino acids. Two missense mutations of V32M and E80X have 
been found in Caucasians (Stambolian et al. 1995). The $G A L K$ genomic gene was mapped to chromosome $17 \mathrm{q} 24$ and consisted of eight exons spanning $7.3 \mathrm{~kb}$ (Bergsma et al. 1996). However, only two GALK-deficient mutations have been reported as published findings.

In this study, we characterized the human $G A L K$ genomic gene from a Japanese genomic DNA library and identified five novel mutations in seven Japanese patients with GALK deficiency.

\section{Patients and methods}

Patients

Seven nonconsanguineous Japanese patients from the main island of Japan were studied. Their biochemical phenotypes and molecular genotypes are presented in Table 1. All seven patients had normal fluorescence in the Beutler screening test for galactose-1-phosphate uridyltransferase (GALT) activity and elevated galactose concentration in the Paigen screening test; the tests were performed at various institutions. The patients were placed on a galactoserestricted diet within the first 2 weeks of life, and were later found to have low levels of GALK activity in erythrocytes at our institution. Patient 4 developed bilateral cataracts, but improved immediately after the introduction of a lactose-free diet. The other patients had no cataracts or other symptoms. Informed consent for genetic analysis was obtained from all subjects or their parents.

Isolation of the human $G A L K$ gene

Total RNA was isolated from cultured human lymphoblasts transformed with Epstein-Barr virus by centrifugation through a $\mathrm{CsCl}$ cushion. For cDNA synthesis, $20 \mu \mathrm{g}$ of total RNA was reverse transcribed using oligo (dT) 12-18 and 30 units of avian myeloblastosis virus reverse transcriptase, as described elsewhere (Kobayashi et al. 1990). The GALK coding region was amplified with primers GALK1 (5'-
AGAGCTGCAGGCGCGCGTCATGGCTGCT-3') and GALK1250 (5'-CGGATATGGAAGATGGCACCGGGCACA-3'), using an LA-PCR Kit (Takara, Otsu, Japan) based on the GALK1 cDNA sequence (Stambolian et al. 1995). PCR products were ligated to the TA cloning vector pT7Blue (Novagen, Madison, WI, USA) and were sequenced to confirm sequence errors.

An EMBL3 library of human genomic DNA from peripheral blood (Japanese Cancer Research Resource Bank, Tokyo, Japan) was screened by in situ plaque hybridization (Benton and Davis 1977) with a randomly ${ }^{32} \mathrm{P}$-labeled probe prepared from a human $G A L K$ cDNA, using the Megaprime DNA labelling system (Amersham Pharmacia Biotech, Uppsala, Sweden). Two independent clones for $G A L K$ genomic DNA were isolated after secondary and tertiary screening, and were cultured to obtain a large amount of genomic DNA. GALK genomic DNA was sequenced by the dye terminator method with a Dye Terminator Cycle Sequencing Ready Reaction Kit (Perkin Elmer, Norwalk, CT, USA) using the Gene Amp 9600 (Perkin Elmer) and an ABI PRISM 310 Genetic Analyzer (Perkin Elmer). Sequencing of $G A L K$ genomes was started with the oligonucleotide primers in $G A L K$ cDNA sequences, followed by the primer sequence walking method. All nucleotides were determined on both coding and non-coding strands.

\section{Identification of human $G A L K$ mutations}

Genomic DNA was prepared from white blood cells or lymphoblasts transformed with Epstein-Barr virus, using a phenol/chloroform extraction method. Control DNA was obtained from phenotypically and biochemically normal adults without a family history of galactosemia. Each exon was subjected to amplification with a pair of human $G A L K$ specific oligonucleotide primers (one primer was biotinylated), using the polymerase chain reaction (PCR). For each reaction, $300 \mathrm{ng}$ of genomic DNA was amplified in a $50-\mu \mathrm{l}$ volume containing $15 \mathrm{pmol}$ of each primer, $1.5 \mathrm{mM}$ $\mathrm{MgCl}_{2}, 0.8 \mathrm{mM}$ dNTPs, $50 \mathrm{mM} \mathrm{KCl}, 10 \mathrm{mM}$ Tris $\cdot \mathrm{HCl}, \mathrm{pH}$ $8.3,0.1 \mathrm{mg} / \mathrm{ml}$ gelatin, and $2.5 \mathrm{U}$ Taq polymerase. Thirty-five

Table 1. Biochemical phenotypes and genotypes of Japanese patients with GALK deficiency

\begin{tabular}{|c|c|c|c|c|}
\hline \multirow[b]{3}{*}{ Patient } & \multirow[b]{3}{*}{ Genotype } & \multicolumn{3}{|l|}{ GALK activity } \\
\hline & & \multicolumn{2}{|c|}{ In patients' erythrocytes ${ }^{a}$} & \multirow{2}{*}{$\begin{array}{l}\text { In COS cell } \\
\text { expression analysis }\end{array}$} \\
\hline & & Aged $<1$ Year & Aged $>1$ Year & \\
\hline 1 & T344M/unknown & & $3.1 \%$ (14 Years) & $1 \% /-$ \\
\hline 2 & G349S/G349S & $6.6 \%$ (5 Months) & $1.0 \%$ (4 Years) & $0 \% / 0 \%$ \\
\hline 3 & R256W/T344M & & $3.3 \%$ (3 Years) & $0 \% / 1 \%$ \\
\hline 4 & 410delG/unknown & $10.2 \%$ (1 Month) & $1.4 \%$ (1 Years) & $0 \% /-$ \\
\hline 5 & 509-510delGT/unknown & $30.0 \%$ (1 Month) & $13.9 \%$ (2 Years) & $0 \% /-$ \\
\hline 6 & $\mathrm{~T} 344 \mathrm{M} / \mathrm{T} 344 \mathrm{M}$ & & $4.8 \%$ (13 Years) & $1 \% / 1 \%$ \\
\hline 7 & G349S/unknown & $2.1 \%$ (1 Month) & & $0 \% /-$ \\
\hline
\end{tabular}

${ }^{a}$ GALK activity is shown as a percentage of the GALK activity in normal adult control

${ }^{\mathrm{b}}$ GALK activity in cells transfected with mutant $G A L K$ cDNA is shown as a percentage of GALK activity in cells transfected with normal $G A L K$ cDNA 
cycles of amplification were carried out with the following thermal profile: denaturation at $94^{\circ} \mathrm{C}$ for $45 \mathrm{~s}$, annealing at $55^{\circ} \mathrm{C}$ for $1 \mathrm{~min}$, and extension at $72^{\circ} \mathrm{C}$ for $1 \mathrm{~min}$. An initial denaturation step of $30 \mathrm{~s}$ at $94^{\circ} \mathrm{C}$ and a final extension step of $2 \mathrm{~min}$ at $72^{\circ} \mathrm{C}$ were added. Each sequence change was identified using the following primer set for PCR amplification: 3-5 (5'-TTCCTGTGCCATCCTCCCAG-3') and $3-3 \quad\left(5^{\prime}\right.$-CCATAAGGCATAGTAGAAGC- $\left.3^{\prime}\right)$ for exon 3 ; $4-5$ (5'-GAATCTCCCTGGAGTGTCATT-3') and 4-3 (5'-CAGGCAGTGGGCACACTCCA-3') for exon 4; 5-5 (5'-TGGAGTGTGCCCACTGCCTG-3') and 5-3 (5'ACAGCCGCCTCCAGGATAGA-3') for exon 5; and 7-5 (5'-CCCAGGCCCACCCCTTCAATA-3') and 7-3 (5'CCCGGGAAGCTGCCGCTCCT-3') for exon 7. The amplified products were purified to single-strand DNA using magnetic beads coated with streptavidin M280 (Dynal, Oslo, Norway). This single-strand DNA was sequenced directly by the dideoxynucleotide chaintermination method, using a Sequenase Version 2.0 DNA Sequence Kit (Amersham Pharmacia Biotech).

\section{Expression analysis}

Mutant human $G A L K$ cDNA was synthesized by specific base substitutions, using site-directed mutagenesis into a eukaryotic expression vector (pCDNA3; Invitrogen, San Diego, CA, USA) containing a full-length human $G A L K$ cDNA. Mutant and wild-type $G A L K$ cDNAs were introduced into COS cells, in a mixture of $20 \mathrm{mM}$ HEPES, $\mathrm{pH}$ $7.05,137 \mathrm{mM} \mathrm{NaCl}, 5 \mathrm{mM} \mathrm{KCl}, 0.7 \mathrm{mM} \mathrm{Na}_{2} \mathrm{HPO}_{4}$, and $6 \mathrm{mM}$ dextrose, by electroporation with a Gene Pulser (BioRad, Hercules, CA, USA) at $200 \mathrm{~V}$ with $960-\mu \mathrm{F}$ capacitance, as described elsewhere (Ashino et al. 1995). The cells were harvested after 72-h culture. GALK activities were determined twice to ensure reproducibility, using ${ }^{14} \mathrm{C}$-galactose with a chromatographic procedure employing a diethylaminoethyl (DEAE)-cellulose column, as described elsewhere (Shin Buehring et al. 1977), and were normalized by relative variations in the levels of $G A L K$ mRNA. GALK mRNA levels in cell extracts were determined by dot-blot hybridization for serially diluted totalRNA samples with a $G A L K$ cDNA probe labeled with $\left[\alpha-{ }^{32} \mathrm{P}\right]$ dCTP (Du-Point-NEN, Boston, MA, USA), using the Megaprime DNA labelling system (Amersham Pharmacia Biotech). The level of GALK activity in cells transfected with mutant $G A L K$ cDNA was expressed as a percentage of that in cells transfected with the normal $G A L K$ cDNA.

\section{Results}

Isolation and characterization of the human $G A L K$ gene

We screened the human genomic DNA phage library in $\lambda$ EMBL3-based vectors, with a human $G A L K$ cDNA probe. Two independent clones ( $\lambda$ HG-GALK1 and $\lambda$ HGGALK9) were finally isolated from $10^{6}$ phage plaques screened initially. $\lambda$ HG-GALK1 contained about $8 \mathrm{~kb}$, and exhibited the structure of the $G A L K$ gene following primer sequence walking. Our $G A L K$ gene was organized into eight exons spanning $7.3 \mathrm{~kb}$, and revealed no differences in coding sequence from the published cDNA sequence (Stambolian et al. 1995). All splice junctions agreed with a previously published description of the human gene (Bergsma et al. 1996). In addition, a 20-bp tandem repeat (ATTCTCCTGCCTCAGCCTCC) was found in three places in intron 5 . Several nucleotides in the $5^{\prime}$-untranslated region (5'-UTR) and introns 1,2 , and 5 in our $G A L K$ genomic analysis differed from those in the published report. Substitutions were found at three places in the 5'UTR, 6 in intron 1, 6 in intron 2, and 28 in intron 5 . Deletions of one nucleotide were found in two locations each in introns 1 and 2. Insertion of one nucleotide was found at one location each in introns 1 and 2 (data not shown: see GenBank homepage under Accession No. AF084935 for details). We confirmed the sequences of $5^{\prime}$ UTR, introns 1 and 2, and a part of intron 5 in three Japanese patients with GALK deficiency, two Caucasian patients with GALK deficiency, and two Japanese wild-type controls. All seven sequences in the $5^{\prime}$-UTR and introns 1 , 2 , and 5 were the same as in our $G A L K$ gene.

\section{Characterization of human $G A L K$ mutations}

We identified five novel mutations, ie, three missense mutations and two deletions, in seven Japanese patients with GALK deficiency (Table 2, Fig. 1). We detected a C-to-T transition at nucleotide position 766 of the GALK genomic gene in exon 5, resulting in the replacement of Arg (CGG) by Trp (TGG) at codon 256 (R256W). The R256W mutation occurred at the $\mathrm{CpG}$ dinucleotide and did not occur in conserved regions. We detected two missense mutations in exon 7: one was a C-to-G transition at nucleotide position 1031 of the $G A L K$ genomic gene, resulting in the replacement of Thr (ACG) by Met (ATG) at codon 344 (T344M). The other was a G-to-A transition at nucleotide position

Table 2. GALK mutations in Japanese patients with GALK deficiency

\begin{tabular}{llllll}
\hline Systematic name & Trivial name & Exon & Codon & CpG & Conserved \\
\hline c.410delG & L135/G136/G137fsdelG & 3 & $(405 \rightarrow 410)$ delG & - & - \\
c.509-510delGT & V169/C170fsdelGT & 4 & $(505 \rightarrow 510)$ delGT & - & - \\
c.766C $\rightarrow \mathrm{T}$ & R256W & 5 & CGG/TGG & Yes & No \\
c.1031C $\rightarrow$ T & T344M & 7 & ACG/ATG & Yes & Yes \\
c.1045G $\rightarrow \mathrm{A}$ & G349S & 7 & GGT/AGT & Yes & Yes \\
\hline
\end{tabular}




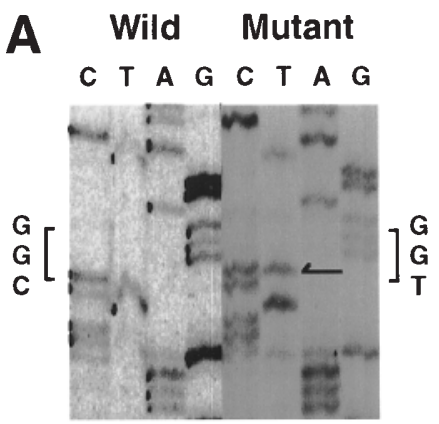

(Exon5)

$\operatorname{Arg}^{256}$

W 5'- CTCCGGGAG -3'

M 5'- - - TGG - - - -

D

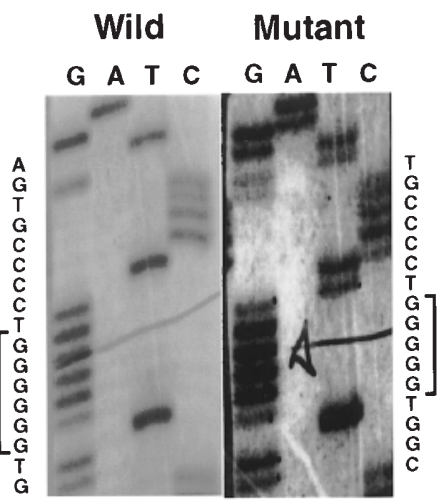

(Exon3)

W 5'- CCCTGGGGGGTG -3'

M 5'- CCCCTGGGGGTG -3'
$B$ Wild Mutant

C $T A G C$ C $A$ G

$A$
$C$
$G$[

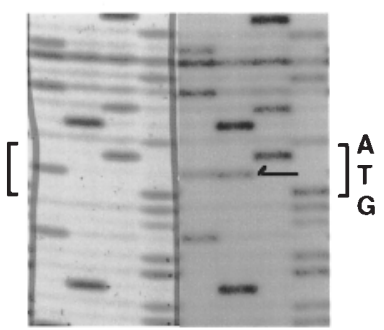

(Exon7)

$\mathrm{Thr}^{344}$

W 5'- ATGACGGGC -3'

M 5' - . - ATG - . - -3'

Met $^{344}$
C Wild Mutant

C T A G C $T$ A G

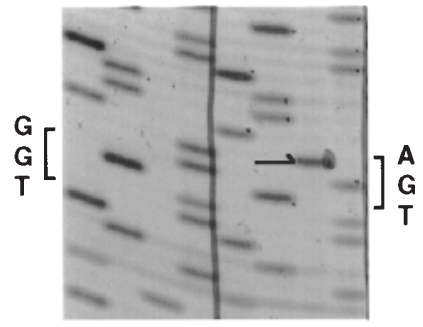

(Exon7)

Gly ${ }^{349}$

W 5' - TTCGGTGGC-3'

M 5' - - - AGT - - - -3'

Ser ${ }^{349}$

E Wild Mutant

G A T C G A T C

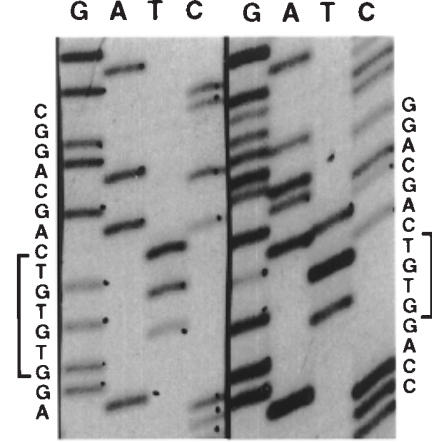

(Exon4)

W 5'- GACGACTGTGTGGA -3'

M 5'- CGGACGACTGTGGA -3'

509-510delGT

Fig. 1A-E. Identification of five novel mutations of the human $G A L K$ gene. A The regions containing exon 5 from a wild-type individual and patient 3 were amplified by polymerase chain reaction (PCR), using primers 5-5 and 5-3. Note $\mathrm{C}$ band and $\mathrm{T}$ band are present at the same position in the mutant sequence (arrow), indicating heterozygosity for mutation. This C-to-T transition at nucleotide 766 of the GALK cDNA results in the replacement of arginine by tryptophan (R256W). B The regions containing exon 7 from a wild-type individual and patient 1 were amplified by PCR, using primers 7-5 and 7-3. Note $\mathrm{C}$ band and $\mathrm{T}$ band are present at the same position in the mutant sequence (arrow), indicating heterozygosity for mutation. This C-to-T transition at nucleotide 1031 of the $G A L K$ cDNA results in the replacement of threonine by methionine (T344M). C The regions containing exon 7 from a wildtype individual and patient 2 were amplified by PCR, using primers 7 5 and 7-3. Only an A band, instead of a G band, is present in the mutant

1045, resulting in the replacement of Gly (GGT) by Ser (AGT) at codon 349 (G349S). T344M and G349S occurred in the conserved region, on the ATP-binding motif, and at $\mathrm{CpG}$ dinucleotides. Furthermore, two deletions were detected: one was a 410delG mutation with deletion of one $\mathrm{G}$ at a position from 405 to 410 (GGGGGG), while the other was a 509-510delGT mutation with deletion of $2 \mathrm{bp}$, GT or TG, at a position from 505 to 510 (GTGTGT). The sequence (arrow), indicating homozygosity for mutation. This G-to-A transition at nucleotide 1045 of the $G A L K$ cDNA results in the replacement of glycine by serine (G349S). D The regions containing exon 3 from a wild-type individual and patient 4 were amplified by PCR, using primers 3-5 and 3-3. Note $\mathrm{G}$ band and $\mathrm{T}$ band are present at nucleotide 405 of the GALK cDNA, while the nucleotide bands above 405 sometimes exhibit double bands in the same positions. This sequence indicates the deletion of $\mathrm{G}$ at nucleotides 405-410. E The regions containing exon 7 from a wild-type individual and patient 5 were amplified by PCR, using primers $4-5$ and $4-3$. Note $\mathrm{G}$ band and $\mathrm{C}$ band are present at nucleotide 509 of the GALK cDNA, while the nucleotide bands above 509 sometimes exhibit double bands at the same positions. This sequence indicates the deletion of GT or TG at nucleotides 505-510. W, Wild; M, Mutant

410delG and 509-510delGT deletions resulted in in-frame nonsense codons at amino acids 163 and 201, respectively.

Expression analysis

To establish that the missense mutations caused galactosemia in our patients, we reconstructed each substitution 
Fig. 2. Analysis of $G A L K$ mRNA in $C O S$ cells transfected with normal or mutant human $G A L K$ cDNA constructs. Dot-blot hybridization for quantitative RNA analysis was performed using the $G A L K$ cDNA as a probe. Serially diluted RNA samples containing 1,2, 4, or $8 \mu \mathrm{g}$ of total RNA extract from transfected COS cells were applied to each lane. GALK activity in cells transfected with mutant $G A L K$ cDNA is shown as a percentage of GALK activity in cells transfected with the normal $G A L K$ cDNA (noted as specific GALK activity, at bottom of Fig.)

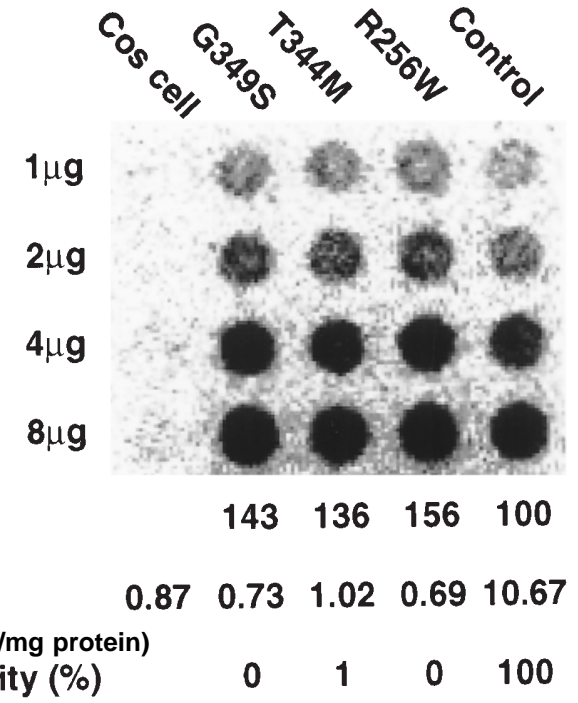

by in-vitro site-directed mutagenesis in the expression plasmid pcDNA3, which allows high-level expression of human $G A L K$ in COS cells. Electroporation of COS cells with wild-type $G A L K$ cDNA in a transient expression assay led to tenfold stimulation of GALK activity over the endogenous background. The GALK activity of each mutated construct was determined by calculating the efficiency of transfection into COS cells; this was done by determining GALK mRNA levels, using the dot-blot hybridization of serially diluted RNA from transfected cells. The GALK activity in all three mutations investigated was decreased to $0-1.2 \%$ of that in the wild-type control, confirming that these mutations caused GALK deficiency (Fig. 2).

\section{Discussion}

We have cloned and sequenced the entire gene for human $G A L K$. Our $G A L K$ gene was organized into eight exons spanning $7.3 \mathrm{~kb}$ and exhibited no differences in coding sequence from the published cDNA sequence (Stambolian et al. 1995). Portions of the sequences in the $5^{\prime}$-UTR and introns 1,2, and 5 differed from the published sequence (Bergsma et al. 1996). We examined these sequences in the $5^{\prime}$-UTR and introns 1, 2, and 5 from five Japanese and two Caucasians. The sequences for all seven subjects were the same as those for our $G A L K$ gene. Since we sequenced the gene for only seven persons, we were unable to determine whether the differences between our findings and published data were due to polymorphisms and/or erros. Interestingly, a 20-bp tandem repeat was found in three places in intron 5. The function and significance of this tandem repeat are unknown. This tandem repeat is frequently present in intronic sequences of genomes in two higher animals, humans and gorillas. We speculate that these sequences were insertion sequences distributed to several sites in higher animals during evolution.

We identified five novel mutations in seven Japanese patients with GALK deficiency. Three missense mutations, of R256W, T344M, and G349S, occurred at CpG dinucleotides, which are mutation hot spots. $\mathrm{CpG}$ dinucleotides within the human coding sequence are up to 42 times more mutable than predicted from random mutation (Cooper and Youssoufian 1988). The 410delG and 509-510delGT deletions occurred at the GGGGGG nucleotide repeat at positions 405 to 410 in exon 3 and at the GTGTGT nucleotide repeat at positions 505 to 510 in exon 4, respectively. Short direct repeats are hot spots for short deletions; in particular, repeats with nucleotide $\mathrm{G}$ cause deletion more frequently than those with other nucleotides (Krawczak and Cooper 1991). Short deletions are caused by slipped strand mispairing, which creates a single-stranded loop, followed by DNA elongation and the formation of a mismatch. The five mutations we reported here were all at mutation hot spots. Two GALK mutations in Caucasians (V32M, E80X) have been published, and confirmed to cause GALK deficiency, by Stambolian et al. (1995). All five of the mutations we detected in Japanese patients differed from the mutations characterized in Caucasians. We speculate, based on our limited data, that the origin of $G A L K$ mutations in Japanese differs from that in Caucasians.

We confirmed that these five novel mutations reduced GALK activity. The T344M and G349S mutations in exon 7 occurred in the ATP binding domain, which is essential for GALK protein activity (Stambolian et al. 1995), and occurred in the conserved sequence in homologous enzymes from Escherichia coli (Debouck et al. 1985), Lactobacillus helveticus (Mollet and Pilloud 1991), Kluyveromyces lactis (Meyer et al. 1991), and Streptomyces lividans (Adams et al. 1988). The R256W mutation did not occur in the conserved sequence. The GALK activities of R256W, T344M, and G349S in COS cell expression analysis were, respectively, $0 \%, 1.2 \%$, and $0 \%$, of that of the wild-type $G A L K \mathrm{cDNA}$ construct. The deletions $410 \mathrm{delG}$ and 509-510delGT resulted in in-frame nonsense codons at amino acids 163 and 201, respectively. These results indicate that these five mu- 
tations drastically reduced GALK activity and caused GALK deficiency. It is known that erythrocyte GALK activity in wild-type controls is two to three times higher in the neonatal period than during adulthood (Segal and Berry 1995). As shown in Table 1 , the GALK activities of three patients (patients 2, 4, and 5) in the neonatal and infantile periods were higher than the GALK activities when the patients were aged over 1 year. These results indicate that GALK activity in subjects with GALK deficiency was agedependent, similar to that in wild-type controls. Patients 2, 3 , and 6 , who were found to have mutations in both alleles, exhibited, respectively, $1.0 \%$ (at 4 years), 3.3\% (at 3 years), and $4.8 \%$ (at 13 years) of wild-type erythrocyte GALK activity. These values were almost consistent with the findings of $0 / 0 \%, 0 / 1 \%$, and $1 / 1 \%$, respectively, for in-vitro GALK activity in COS cell expression analysis. Since all these mutations resulted in $0-1 \%$ of wild-type in-vitro GALK activity and a related severe phenotype, it is difficult to determine the relationship between erythrocyte GALK activity and genotype based on GALK activity in COS cell expression analysis. Patient 5, with 509-510delGT and unknown mutations still exhibited a high residual GALK activity, of $13.9 \%$ of the wild-type level in erythrocytes at the age of 2 years. Future studies of $G A L K$-deficient mutations, including this unknown mutation, will address the relationships among genotype, cataract formation, and biochemical phenotype.

Acknowledgments We thank Dr. A. Ohtake, Saitama Medical School, Dr. Y. Wada, Nagoya City University Medical School, Dr. Y. Hasegawa, School of Medicine, Keio University, and Dr. M. Sasaki, Kihoku Hospital, Wakayama Medical College, for providing blood samples from patients with GALK deficiency.

This study was supported in part by a grant from the Ministry of Education, Science, and Culture of Japan. The GALK sequence obtained has been submitted to GenBank under Accession No. AF084935.

\section{References}

Adams CW, Fornwald JA, Schmidt FJ, Rosenberg M, Brawner ME (1988) Gene organization and structure of the Streptomyces lividans gal operon. J Bacteriol 170:203-212
Aoki K, Wada Y (1988) Outcome of the patients detected by newborn screening in Japan. Acta Paediatr Jpn 30:429-434

Ashino J, Okano Y, Suyama I, Yamazaki T, Yoshino M, Furuyama J, Lin HC, Reichardt JK, Isshiki G (1995) Molecular characterization of galactosemia (type 1) mutations in Japanese. Hum Mutat 6:36-43

Benton WD, Davis RW (1977) Screening $\lambda$ gt recombinant clones by hybridization to single plaques in situ. Science 196:180-182

Bergsma DJ, Ai Y, Skach WR, Nesburn K, Anoia E, Van Horn S, Stambolian D (1996) Fine structure of the human galactokinase GALK1 gene [letter]. Genome Res 6:980-985

Cooper DN, Youssoufian H (1988) The CpG dinucleotide and human genetic disease. Hum Genet 78:151-155

Debouck C, Riccio A, Schumperli D, McKenney K, Jeffers J, Hughes C, Rosenberg M, Heusterspreute M, Brunel F, Davison J (1985) Structure of the galactokinase gene of Escherichia coli, the last (?) gene of the gal operon. Nucleic Acids Res 13:1841-1853

Gitzelmann R (1965) Deficiency of erythrocyte galactokinase in a patient with galactose diabetes. Lancet II:670-671

Kobayashi K, Jackson MJ, Tick DB, O’Brien WE, Beaudet AL (1990) Heterogeneity of mutations in argininosuccinate synthetase causing human citrullinemia. J Biol Chem 265:11361-11367

Krawczak M, Cooper DN (1991) Gene deletions causing human genetic disease: mechanisms of mutagenesis and the role of the local DNA sequence environment. Hum Genet 86:425-441

Litman N, Kanter AI, Finberg L (1975) Galactokinase deficiency presenting as pseudotumor cerebri. J Pediatr 86:410-412

Magnani M, Cucchiarini L, Dacha M, Fornaini G (1982) A new variant of galactokinase. Hum Hered 32:329-334

Meyer J, Walker Jonah A, Hollenberg CP (1991) Galactokinase encoded by $G A L 1$ is a bifunctional protein required for induction of the GAL genes in Kluyveromyces lactis and is able to suppress the gal3 phenotype in Saccharomyces cerevisiae. Mol Cell Biol 11:54545461

Mollet B, Pilloud N (1991) Galactose utilization in Lactobacillus helveticus: isolation and characterization of the galactokinase ( $\mathrm{galK}$ ) and galactose-1-phosphate uridyl transferase (galT) genes. J Bacteriol 173:4464-4473

Segal S, Berry G (1995) Disorders of galactose metabolism. In: Scriver C, Beaudet A, Sly W, Valle D (eds) The metabolic and molecular bases of inherited disease, 7th edn. McGraw-Hill, New York, pp 967-1000

Segal S, Rutman JY, Frimpter GW (1979) Galactokinase deficiency and mental retardation. J Pediatr 95:750-752

Shin Buehring YS, Osang M, Ziegler R, Schaub J (1977) A simple assay for galactokinase using DEAE-cellulose column chromatography. Clin Chim Acta 74:1-5

Stambolian D (1988) Galactose and cataract. Surv Ophthalmol 32:333-349

Stambolian D, Ai Y, Sidjanin D, Nesburn K, Sathe G, Rosenberg M, Bergsma DJ (1995) Cloning of the galactokinase cDNA and identification of mutations in two families with cataracts. Nat Genet 10:307-312

Tedesco TA, Miller KL, Rawnsley BE, Adams MC, Markus HB, Orkwiszewski KG, Mellman WJ (1977) The Philadelphia variant of galactokinase. Am J Hum Genet 29:240-247 